\title{
COMMENTARY
}

\section{Focusing light: still full of surprises}

\author{
Maximilian Kreiter \\ Max-Planck Institut für Polymerforschung, Ackermannweg 10, 55128 Mainz, Germany \\ kreiter@mpip-mainz.mpg.de
}

The basic theoretical concepts behind the tight focusing of light beams were essentially developed in the 19th century and have been regarded as common knowledge for many decades [1]. In the last ten years, the increased need to optically access individual organic dyes or nanostructures has led to a renewed interest in the field. Novel experimental schemes have been proposed and implemented to take advantage of a large flexibility to optimally tailor electromagnetic field distributions in the focal region of a microscope objective to different experimental needs. This approach of focal engineering is the key to several scientific discoveries.

Generally, the field in the focal region of a microscope objective illuminated by a light field propagating parallel to the optical axis can be regarded as the superposition of plane light waves originating from directions which are determined by the objective's numerical aperture. This concept is depicted schematically in Fig. 1(a). A large variety of focal field distributions can be obtained by controlling each of these plane-wave contributions in the back focal plane of the objective. With modern LCD technology, almost no restrictions for the manipulation of their intensity and polarization state exist.

Already, simple incident light fields yield rich and useful focal patterns. Uniform intensity and fixed linear polarization state gives rise to a three-dimensional (3D) field distribution as depicted in Fig. 1(b). The electric field's component parallel to the incident light's polarization direction $\left(\mathrm{E}_{\mathrm{x}}\right)$ dominates and its distribution is similar to the Airy pattern that would be obtained in the paraxial approximation. In addition, a smaller but sizable contribution aligned along the propagation direction $\left(\mathrm{E}_{\mathrm{z}}\right)$ as well as a contribution perpendicular to the incident light's polarization and propagation directions $\left(\mathrm{E}_{\mathrm{y}}\right)$ exist. The appearance of the latter two components, which are orthogonal to the polarization direction of the incident light, can be understood by following the rays that change their propagation direction in the focal sphere. Each pattern has a characteristic symmetry and distribution of nodal planes.

A single organic dye molecule which possesses a transition dipole moment that is fixed in space will experience a driving electric field corresponding to the projection of the 3D field on this direction. Sampling the dye excitation rate pixel by pixel, while scanning through the focal region, yields a coherent superposition of the three patterns corresponding to $\mathrm{x}-, \mathrm{y}-$ and z-polarization states. Blocking of the part of the incident light close to the optical axis by placing an absorbing disk in the center of the back focal plane decreases the differences in maximum field amplitudes between the three Cartesian components and allows for a reliable determination of the 3D orientation of the dye molecule [2]. This approach to determine molecular orientations and follow them in time has become an accepted experimental method. It is used to address various scientific questions, e.g. the molecular-scale dynamics in polymers [3].

A radially polarized field in the back focal plane represents another example for a simple incident light field with interesting properties: Dorn et al. demonstrated that it gives rise to focal sizes which are smaller than with linearly polarized (or unpolarized) light with a corresponding increase in resolution [4]. 

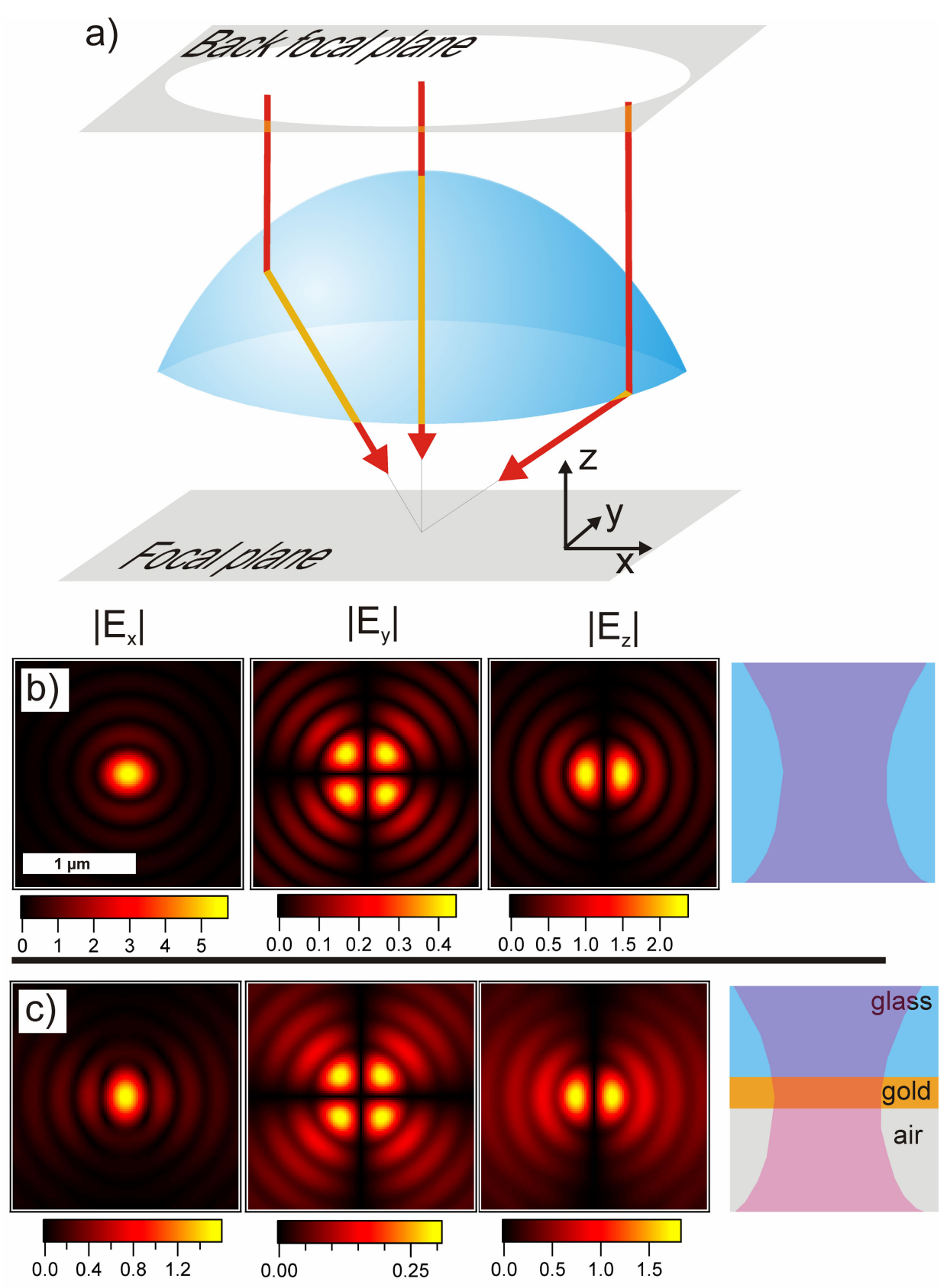

Fig. 1. (a) Simplified illustration of formation of the field distribution within the focal region. Three parallel rays are selected to exemplify. They start at the back focal plane, are transferred to the surface of the focal sphere, and are superimposed in the focal plane. (b) Field amplitudes when focusing linearly (x-) polarized light $(\lambda=633 \mathrm{~nm})$ with an oil-immersion objective into a bulk dielectric material. Note the different scales that had to be chosen. (c) Field amplitudes when focusing through a 50-nm-thick gold film separating glass and air. 
A particularly successful application of well-controlled focal field distributions is known as stimulated emission depletion (STED) microscopy [5]. This technique yields an optical resolution of the order of a 20th of a wavelength. Here, initially dye molecules are excited by a standard diffraction-limited spot. By stimulated emission, a second light pulse brings all molecules that are exposed to its electric field back to the ground state. Only molecules which do not experience that field stay in the excited state. As a consequence, regions with vanishing or very low fields within the focal region become very important. Within the 3D field distribution, true 2D nodal planes with vanishing field components exist. In Fig. 1(b) they are seen for the $y$ - and z-component as dark lines. For high-resolution imaging, an important difference in the lateral definitions of field maxima and nodal planes comes into play: Field maxima have a typical size - e.g. defined as full-width at half maximum - which is always of the order of the wavelength of the incident light. Nodal planes, on the contrary, are strictly 2D entities embedded in 3D space. Therefore, by correctly choosing the intensity threshold, the molecules which remain excited after the second pulse can be confined to arbitrarily small distances to the nodal plane, thereby resulting in the high lateral resolution of STED. Today, this method has made its way to application and is used for imaging of biological samples with previously unthinkable resolution. Recently, this technique shed light on the longstanding question of the transient formation of nanometer-sized domains (rafts) in lipid bilayers, demonstrating how useful well-controlled electromagnetic field distributions within an optical focal region can be [6].

If light is focused at an interface, or more generally on a layered system, the field in the focal region changes significantly. This again can be rationalized in the picture of a superposition of plane waves incident from different directions, see Fig. 1 (a). Depending on angle of incidence and polarization state, each incident plane wave is partially reflected and partially transmitted. Finally, incident, reflected and transmitted waves have to be summed up to obtain the total focal field in the presence of an interface.

A particularly strong angular dependence in the total focal field arises when focusing with an oil-immersion objective through a gold film with a thickness of about $50 \mathrm{~nm}$. In this configuration, a plane wave incident at a certain angle relative to the surface normal gives rise to an electric field at the backside of the metal film which is significantly higher in magnitude than the electric field of the incident plane wave. This field enhancement is attributed to the excitation of surface plasmons on the backside of the gold film. At the same time, the contributions from plane waves incident from all other directions which do not lead to the excitation of surface plasmons are strongly suppressed. As a consequence, the multilayer system effectively selects only a small fraction of the incident light field, corresponding to a cone of incident light rays or a ring in the back focal plane. The resulting field, as shown in Fig. 1(c), is dominated by the longitudinal (z-) component. Intensity patterns cover larger areas than in the absence of the interface, as can be understood as an effectively decreased numerical aperture.

Such patterns have been observed experimentally in our group by single-molecule fluorescence [7] and by Bouhelier et al. by scanning near-field optical microscopy [8]. This restricted angular regime selected by the multilayer system is of great importance for darkfield microscopy through thin metal films. Any blocking scheme with circularly symmetric blocks that preserves the symmetry of the optical system only yields very weak signals. In an asymmetric blocking scheme, the reduced effective area in the back focal plane is taken into account and sensitive measurements of scattering efficiencies are possible, taking advantage of the intermediate excitation of surface plasmons [9].

In summary, the use of the full 3D vector character of light in the focal region of highnumerical aperture objectives has been exemplified. Relatively straightforward modifications of the incident light made the optical microscope an even more useful instrument than it has been for centuries. I expect that the choices for incident fields which are based on an educated guess will be replaced by the results of systematic optimization routines for specific 
experimental needs in the future. Tailored incident light fields will allow for previously unknown control over the electromagnetic field. Additional degrees of freedom arise if focal engineering is combined with control over the time-dependent optical field of ultrashort laser pulses. In such a scheme, almost arbitrary evolutions of the 3D electromagnetic field in time could be envisaged.

\section{References}

[1] M. Born and E. Wolf, Principles of Optics, $7^{\text {th }}$ ed., Cambridge University Press, Cambridge, UK(2006).

[2] B. Sick, B. Hecht, and L. Novotny, "Orientational imaging of single molecules by annular illumination," Phys. Rev. Lett. 85, 4482-4485 (2000) [doi:10.1103/PhysRevLett.85.4482].

[3] R. A. L. Vallee, M. Van der Auweraer, W. Paul, and K. Binder, "What can be learned from the rotational motion of single molecules in a polymer melt near the glass transition?" Europhys. Lett. 79, 46001 (2007) [doi:10.1209/02955075/79/46001].

[4] R. Dorn, S. Quabis, and G. Leuchs, "Sharper focus for a radially polarized light beam," Phys. Rev. Lett. 91, 233901 (2003) [doi:10.1103/PhysRevLett.91.233901].

[5] V. Westphal and S. W. Hell, "Nanoscale resolution in the focal plane of an optical microscope," Phys. Rev. Lett. 94, 143903 (2005) [doi:10.1103/PhysRevLett.94.143903].

[6] C. Eggeling, C. Ringemann, R. Medda, G. Schwarzmann, K. Sandhoff, S. Polyakova, V. N. Belov, B. Hein, C. von Middendorff, A. Schönle, and S. W. Hell, "Direct observation of the nanoscale dynamics of membrane lipids in a living cell," Nature 457, 1159-1162 (2009) [doi:10.1038/nature07596].

[7] F. D. Stefani, K. Vasilev, N. Bocchio, N. Stoyanova, and M. Kreiter, "Surfaceplasmon-mediated single-molecule fluorescence through a thin metallic film," Phys. Rev. Lett. 94, 023005 (2005) [doi:10.1103/PhysRevLett.94.023005].

[8] A. Bouhelier, F. Ignatovich, A. Bruyant, C. Huang, G. Colas des Francs, J.-C. Weeber, A. Dereux, G. P. Wiederrecht, and L. Novotny, "Surface plasmon interference excited by tightly focused laser beams," Opt. Lett. 32, 2535-2537 (2007) [doi:10.1364/OL.32.002535].

[9] M. Schmelzeisen, M. J. Austermann, and M. Kreiter, "Plasmon mediated confocal dark-field microscopy," Opt. Exp. 16, 17826-17841 (2008) [doi:10.1364/OE.16.017826]. 